\title{
EVALUACIÓN DE TRES MÉTODOS PARA EL ARRASTRE DE MADERA EN RODALES NATURALES DE Pinus caribaea var. caribaea $^{1}$
}

Fidel Cándano Acosta², Alberto Vidal Corona ${ }^{3}$, Angelo Márcio Pinto Leite ${ }^{4}$, Carlos Cardoso Machado ${ }^{5}$

\begin{abstract}
RESUMEN - El trabajo se realizó en rodales naturales de Pinus caribaea var. caribaea, ubicados en la unidad silvícola San Andrés, La Palma, provincia Pinar del Río. El objetivo de la investigación fue evaluar la eficiencia y los daños causados a la cubierta vegetal del suelo durante la operación de arrastre de madera. Se evaluaron tres métodos: 1 - el arrastre de fustes enteros con yuntas de bueyes hasta los patios de carga; 2 - el arrastre de fustes enteros con tractor hasta los patios de carga; 3 - el arrastre de fustes enteros con yuntas de bueyes hasta las pistas de arrastre y con tractor hasta los patios de carga. El método de arrastre de madera que consistió en la combinación del empleo de animales y de tractor resultó más eficiente y provocó menos alteraciones a la cubierta vegetal del suelo.
\end{abstract}

Palabras-claves: Métodos de arrastre; rendimientos; costos y daños a la cubierta vegetal.

\section{EVALUATION OF THREE METHODS OF THE LOG Pinus caribaea SKIDDING IN NATURAL SET OF WHEELS}

\begin{abstract}
The work was carried out in natural stand of Pinus caribaea var. caribaea located in San Andrés, Forestry enterprise of La Palma in the Pinar del Río province. The objective was determine which method of wood extraction results more efficient and reduce damages to the soil vegetable. Three method were evaluated: 1- the extraction of tree length with oxen to landing; 2 - the extraction of tree length with tractor to landing; 3 - the extraction of tree length with oxen to short distance and using tractor up to landing places. The method the extraction of tree length with oxen to short distance and using tractor up to landing places, resulted the most efficient and the one which fewer damages on the vegetable cover of the soil.
\end{abstract}

Key words: Extraction method, yields, costs and disturbance.

\section{INTRODUCCIÓN}

La empresa forestal "La Palma", ubicada en la Provincia de Pinar del Río, cuenta con cerca de 2 mil hectáreas de pinares nativos explotados, de donde se extraen de 60 a 80 metros cúbicos de madera diarios para abastecer a dos aserraderos instalados. De acuerdo con Minagri (1997), se proyecta la construcción de un nuevo aserradero, con el cual aumentarán considerablemente los volúmenes de madera a cosechar.

Desde 1981, los tractores de orugas han sido los más utilizados para el arrastre de madera en estos bosques, sin embargo, hay que destacar algunos aspectos negativos de esta tecnología:

- El bajo rendimiento de estos tractores, debido al alto gasto de tiempo en el amarre de la carga, lo

\footnotetext{
${ }^{1}$ Recebido para publicação em 12.11.2002 e aceito para publicação em 08.6.2004.

${ }^{2}$ Prof. de la Universidad de Pinar del Río, Cuba. E-mail: <fcandano@af.upr.edu.cu>.

${ }^{3}$ Instituto de Investigaciones Forestales, Habana, Cuba.

${ }^{4}$ Prof. Faculdades Federais Integradas de Diamantina (FAFEID-DEF), Brasil, E-mail: <ampleite@ig.com.br>.

${ }^{5}$ Prof. de la Universidade Federal de Viçosa (UFV-DEF), Brasil. E-mail: <machado@ufv.br>.
} 
cual resulta de las pequeñas dimensiones de la madera cosechada y de los bajos volúmenes por hectárea.

- La baja eficiencia energética de los motores de tractores de oruga, en comparación a otros tipos de tractores (HARVEY, 1986).

- Los elevados daños a la cubierta vegetal cuando el tractor trabaja fuera de las vías de saca, sobre todo en las maniobras de viraje.

El arrastre de madera es una de las operaciones más costosas de la cosecha forestal, según los resultados obtenidos por Sessions (1992), Seixas et al. (1996) y Volpato et al. (1991). La utilización de tractores para el arrastre de madera es la tecnología más difundida por todo el mundo. Se caracteriza por altos rendimientos y bajos costos cuando comparada a otros métodos, aunque provoca daños considerables al suelo, conforme han demostrado los resultados publicados por Hendrison (1990), Warkotsch y Olsen (1992), Sarre (1993) y Fenner (1996).

El uso intensivo de tractores para la extracción de madera en Cuba está limitado por la falta de financiamiento para adquirirlos y mantenerlos. Además, esta tecnología causaría la intensificación del deterioro al suelo, lo cual consecuentemente, afectaría el crecimiento de las futuras plantaciones. Por otra parte, el uso único de animales como alternativa no es factible, pues las empresas no cuentan con la cantidad de animales para arrastrar los volúmenes requeridos de madera. Los obreros que tienen animales de su propiedad prefieren realizar trabajos agrícolas menos peligrosos y más cómodos, además la tecnología basada en animales provocaría mayor densidad de caminos forestales y patios de carga aumentando los impactos al suelo.

En concordancia con Chirgwin (1994), los esfuerzos actuales por promover el uso de animales de trabajo no pretenden sustituir a la maquinaria motorizada, sino más bien, fomentar una complementariedad entre sus funciones. Por esto, este trabajo tiene como propósito evaluar la eficiencia y los daños a la cubierta vegetal del suelo proporcionados por tres métodos para el arrastre de madera:

- El arrastre de fustes enteros con yuntas de bueyes hasta los patios de carga.

- El arrastre de fustes enteros con tractor hasta los patios de carga.

- El arrastre de fustes enteros con yuntas de bueyes hasta las pistas de arrastre y con tractor hasta los patios de carga.

\section{MATERIALES Y METODOS}

El estudio se realizó en rodales naturales de Pinus caribaea var. caribaea, en la provincia de Pinar del Río, entre los meridianos $22^{\circ} 20^{\prime}$ y $22^{\circ} 45^{\prime}$ de latitud Norte y $83^{\circ} 50^{\prime}$ y $84^{\circ} 27^{\prime}$ de longitud Oeste. El diámetro promedio de los árboles fue de $22.6 \mathrm{~cm}$, la altura de $14.8 \mathrm{~m}$. El volumen promedio de estos fue $0.25 \mathrm{~m}^{3}$ y el volumen por hectárea $80 \mathrm{~m}^{3}$.

De acuerdo con el INRH (1996), la temperatura media anual del sitio es de $23.8^{\circ} \mathrm{C}$, la humedad relativa de $74 \%$, la precipitación de $1686.6 \mathrm{~mm}$. El terreno, ubicado a una altura de 127 metros sobre el nivel del mar (msnm), tiene un relieve ondulado, con pendientes entre 12 y 25\%. El suelo es poco profundo, pobre, con bajo contenido de materia orgánica, clasificado como loam arenoso (HERNÁNDEZ et al., 1994).

Para los tres métodos de arrastre de madera se evaluaron los rendimientos y los costos utilizando los procedimientos metodológicos propuestos en la NORMA CUBANA 34-38 (1985) y Sessions (1992). El volumen de madera se calculó según Grá et al. (1990), después de tomar el diámetro y la longitud de los árboles.

También se estimaron las alteraciones ocasionadas a la cubierta vegetal del suelo por la aplicación de los tres métodos. Tomando como referencia metodológica la investigación de Warkotsch y Olsen (1992), se determinó la magnitud de las alteraciones después de concluir el arrastre de madera en las áreas, expresado en porcentaje por hectárea. Se consideraron como daños severos, aquellos que, además de eliminar la cubierta vegetal, dañaron la parte mineral del suelo.

Se evaluaron 5 yuntas de bueyes que tenían, en promedio, 6 años de edad y más de 1 año de trabajo en las operaciones forestales. El peso de cada animal estuvo comprendido entre 400 y $529 \mathrm{~kg}$. Se utilizó el yugo doble de cabeza que es el tradicional en Cuba y la cadena para el amarre de la madera.

El tractor utilizado fue el TDT-55 de orugas, importado de Rusia, con potencia nominal de 61.91 $\mathrm{kW}$, fuerza de tracción al winche de $66.4 \mathrm{kN}$, velocidad de traslación entre 2.89 y $12.8 \mathrm{~km} / \mathrm{h}, 60$ m de capacidad 
del cable, peso de $9300 \mathrm{~kg}$. Equipado y cargado, el tractor pasa a pesar $10642.8 \mathrm{~kg}$. El área de apoyo del tractor es de $22680 \mathrm{~cm}^{2}$. La presión de contacto con el suelo es de $0.47 \mathrm{~kg} / \mathrm{cm}^{2}$. El equipo de trabajo fue conformado por un operador y dos ayudantes.

\section{RESULTADOS}

\subsection{Análisis del tiempo del ciclo de trabajo de las tecnologías evaluadas}

En la Tabla 1 se aprecia que, para una distancia promedio de $100 \mathrm{~m}$, el método 1 consumió un tiempo de $8.16 \mathrm{~min}$, el cual fue menor que el requerido por el método 2 (14.15 min), a pesar de que los animales desarrollaron una velocidad promedio de $32.55 \mathrm{~m} / \mathrm{min}$ y los tractores de 62.3 y $55.1 \mathrm{~m} / \mathrm{min}$.

La Tabla 1 muestra que el tiempo del ciclo de los animales fue menor que el tiempo consumido por los tractores, lo que parece contradictorio al comparar la velocidad de recorrido de los animales y la de los tractores citadas anteriormente.

La Tabla 1 registra otros elementos que intervinieron en el ciclo de trabajo durante el arrastre de madera, como los tiempos empleados para la carga y descarga del paquete de árboles. Estos datos tienen gran repercusión dentro del ciclo de trabajo, sobre todo para los tractores, cuyos porcentajes de tiempo de carga y descarga fueron de 50.81 y $24.24 \%$ respectivamente, mientras que para los animales, estos porcentajes fueron muy bajos, 12.25 y $11.27 \%$ respectivamente, lo que explica este comportamiento.

La curva que representa el tiempo del ciclo de los tractores en función de las distancias fue menos pronunciada debido a la gran influencia ejercida por el tiempo de carga y descarga de los paquetes de árboles. A diferencia, los animales consumieron mayor cantidad de tiempo en el recorrido que en la carga y descarga, lo que explica la tendencia a incrementar aceleradamente el tiempo del ciclo en función de las distancias.

La Tabla 2 registra el desglose del tiempo de carga requerido para cada tecnología considerada.

Los animales extrajeron, en promedio, 2.09 árboles por ciclo, consumiendo 1.92 min en la carga y descarga, mientras que, en esta operación, los tractores requirieron $10.62 \mathrm{~min}$, de los cuales, $7.19 \mathrm{~min}$ fueron gastos en la carga, como se aprecia en la Tabla 1. La distribución del tiempo de carga se encuentra desglosada en la Tabla 2, donde se observa que fueron requeridos 1.28 min para estirar el cable, 3.28 min en el amarre de los árboles y 2.62 min para la operación

Tabla 1 - Resultados de los indicadores del ciclo de trabajo Table 1 - Results of the work cycle indicators

\begin{tabular}{|c|c|c|c|c|c|c|}
\hline \multirow{2}{*}{$\begin{array}{l}\text { Elementos del tiempo del ciclo } \\
\text { (min) }\end{array}$} & \multicolumn{2}{|c|}{$\begin{array}{l}\text { Yunta de bueyes } \\
\text { (método 1) }\end{array}$} & \multicolumn{2}{|c|}{$\begin{array}{l}\text { Tractores } \\
\text { (método 2) }\end{array}$} & \multicolumn{2}{|c|}{$\begin{array}{c}\text { Bueyes + Tractores } \\
\text { (método 3) }\end{array}$} \\
\hline & 2.71 & $(33.21 \%)$ & 1.66 & $(11.73 \%)$ & 1.52 & $\overline{(13.97 \%)}$ \\
\hline Tiempo de carga & 1.00 & $(12.25 \%)$ & 7.19 & $(50.81 \%)$ & 4.11 & $(37.78 \%)$ \\
\hline Tiempo de recorrido con carga & 3.53 & $(43.26 \%)$ & 1.87 & $(13.26 \%)$ & 1.83 & $(16.81 \%)$ \\
\hline Tiempo de descarga & 0.92 & $(11.27 \%)$ & 3.43 & $(24.24 \%)$ & 3.44 & $(31.62 \%)$ \\
\hline Tiempo total del ciclo & 8.16 & $(100 \%)$ & 14.15 & $(100 \%)$ & 10.88 & $(100 \%)$ \\
\hline Distancia de extracción (m) & 100 & & 100 & & 100 & \\
\hline Distancia operac.winche (m) & & & 17.33 & & 7.96 & \\
\hline Número de árboles por ciclo & 2.09 & & 9.2 & & 10.3 & \\
\hline Volumen por ciclo $\left(\mathrm{m}^{3}\right)$ & 0.36 & & 2.25 & & 2.58 & \\
\hline
\end{tabular}

Tabla 2 - Distribución del tiempo de carga para cada tecnología Table 2 - Loading time distribution for each technology

\begin{tabular}{|c|c|c|c|c|}
\hline Elementos del tiempo del ciclo (min) & Yunta de bueyes & Tractores & \multicolumn{2}{|c|}{ Bueyes + Tractores } \\
\hline Tiempo de movimiento sin carga del winche & & $(17.8 \%)$ & 0.83 & $(20.2 \%)$ \\
\hline Tiempo de amarre de los árboles & 1.00 & $(45.6 \%)$ & 2.15 & $(52.3 \%)$ \\
\hline Tiempo de movimiento con carga del winche & & $(36.4 \%)$ & 1.12 & $(27.3 \%)$ \\
\hline Tiempo total de carga & & $(100 \%)$ & 4.10 & $(100 \%)$ \\
\hline
\end{tabular}


con el winche hasta colocar los fustes en posición de transporte sobre el tractor. Estos resultados se derivan de una distancia promedio con el winche de $17.33 \mathrm{~m}$, dada por la separación entre los árboles derribados, con un promedio de 9.2 troncos por ciclo para completar la carga.

El empleo del método 3, que consistió en hacer los paquetes utilizando los animales previamente (Figura 1) y, extraer la madera hasta los patios de carga con los tractores (Figura 2), posteriormente, logró reducir el tiempo de carga de la madera para el tractor y evitar el movimiento de los animales a larga distancia. Por ello, los resultados de esta variante, según la Figura 1, muestran el punto crítico del tiempo del ciclo a $150 \mathrm{~m}$ aproximadamente y no a $200 \mathrm{~m}$, como cuando se utilizaron los métodos 1 y 2 . Con esta combinación se logró reducir el tiempo del ciclo del tractor de $14.15 \mathrm{~min}$ a $10.88 \mathrm{~min}$, con la mayor variación para el tiempo de carga, que pasó de $50.81 \%$ a $37.78 \%$, dentro del tiempo total del ciclo, resultado de la disminución brusca de la distancia de operación con el winche de $17.33 \mathrm{~m}$ a $7.96 \mathrm{~m}$ y el agrupamiento de los árboles, lo cual redujo el tiempo carga (Tabla 2).

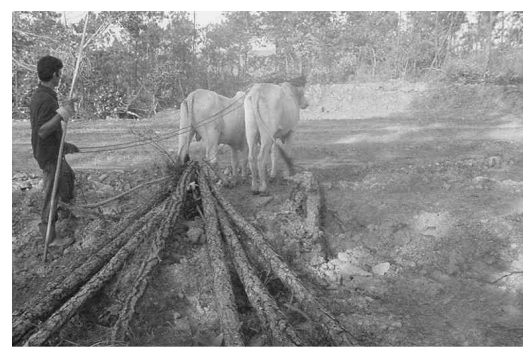

A

\subsection{Análisis de los costos de las variantes}

La Figura 3 muestra que el uso de animales para el arrastre de madera fue más económico que la utilización de los tractores, hasta una distancia aproximada de $200 \mathrm{~m}$. La combinación de animales y tractores, para cualquier distancia, mostró un menor costo que cuando se emplean solamente tractores y el punto critico de la utilización de animales y de tractores se obtuvo a una distancia de $150 \mathrm{~m}$ aproximadamente.

La economía verificada en la operación que utilizó animales no se basó en los altos rendimientos, sino en los bajos costos de la explotación, que fueron del orden de 1.25 pesos por hora, mientras que para la utilización de tractores este valor fue de 7.87 pesos por hora (Tabla 3). La disminución del costo unitario (Figura 4) en el método 3 con respecto al método 2, se explica en el aumento significativo del rendimiento en el método 3.

Aunque el costo del arrastre de madera con animales fue inferior al costo de la misma operación realizada con tractores, la utilización de los animales para el arrastre de madera podría acarrear otras consecuencias que posiblemente tornarían más caro el aprovechamiento de forma general.

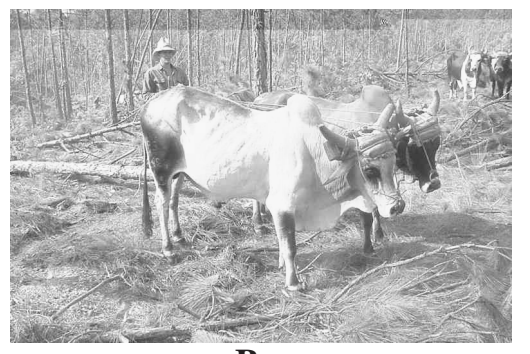

B

Figura 1 - A) Yunta de bueyes arrastrando un paquete de madera. B) Detalle del yugo de cabeza. Figure 1 - a) Ox yoke pulling wood. b) Detail of the head Yoke.

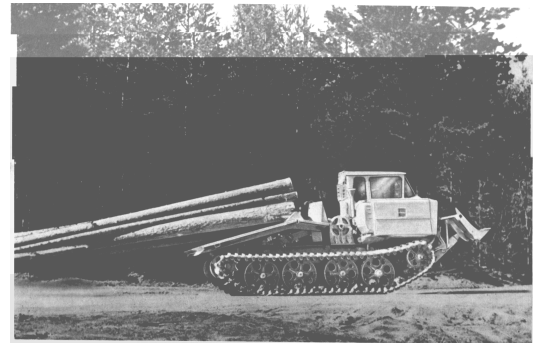

A

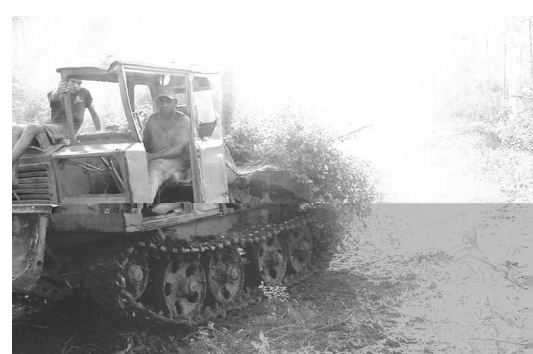

B

Figura 2 - A) Vista general del tractor TDT-55. B) Muestra del sistema de rodaje

Figure $2-$ a) General view of the TDT-55 tractor. b) General view of the set Wheel system 


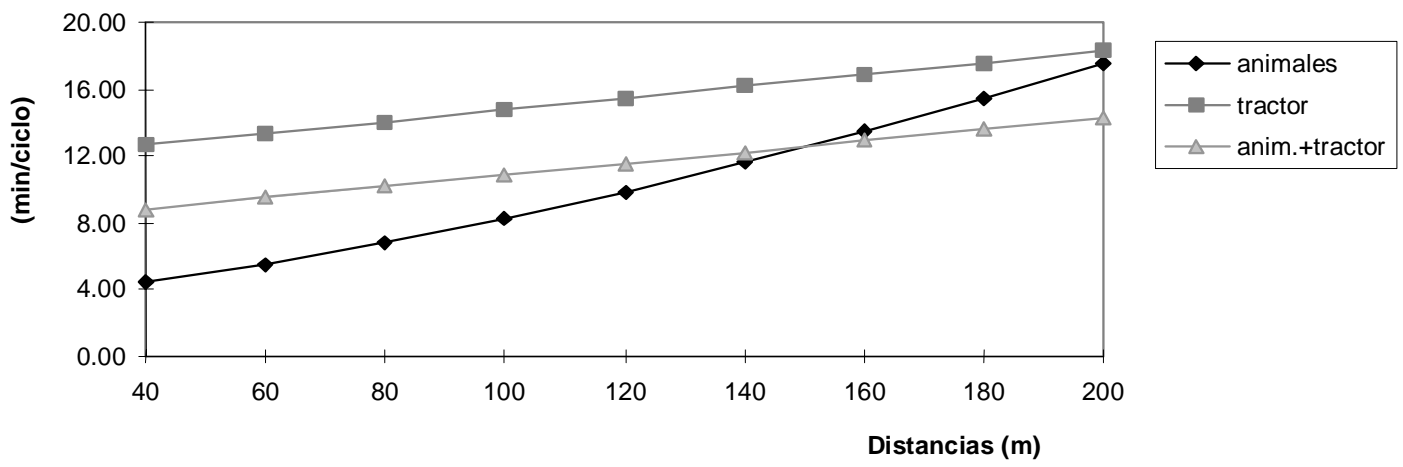

Figura 3 - Tiempos del ciclo en función de las distancias de arrastre.

Figure 3 -Cycle times as function of the pulling distances.

Asumiendo distancias menores de $150 \mathrm{~m}$, quedarían abiertas las preguntas: ¿Qué densidad de camino habría que asumir? ¿Qué distancia entre puntos de carga resultaría factible y cuál sería el costo de construcción de caminos y patios de carga?

El uso sólo de tractores podría disminuir los costos de construcción de caminos y patios de carga, pero implicaría un mayor costo en la operación de arrastre. Esto hace evidente que la mayor eficiencia del tractor para este tipo de bosque se obtuvo cuando se combinó el arrastre previo de la madera con los animales a cortas distancias.

Desde el punto de vista de la eficiencia energética, el método 3 implicó en un ahorro de 0.4 litro de combustible por cada metro cúbico extraído, cuando comparado con el método 2. Esto significaría un ahorro de alrededor de 30 litros diarios, considerando una
Tabla 3 - Resultados de los indicadores principales de cada método

Table 3 - Main results of indicators for each method

\begin{tabular}{|c|c|c|c|}
\hline Indicadores & $\begin{array}{l}\text { Yunta de } \\
\text { bueyes }\end{array}$ & Tractores & $\begin{array}{l}\text { Bueyes + } \\
\text { tractores }\end{array}$ \\
\hline $\begin{array}{l}\text { Costo de explotación } \\
(\$ / \mathrm{h})\end{array}$ & $1.25 *$ & 7.87 & 9.25 \\
\hline Rendimiento (m³/h) & 2.65 & 9.54 & 14.65 \\
\hline Costo unitario $\left(\$ / \mathrm{m}^{3}\right)$ & 0.47 & 0.83 & 0.57 \\
\hline $\begin{array}{l}\text { Consumo de } \\
\text { combustible }\left(1 / \mathrm{m}^{3}\right)\end{array}$ & & 1.5 & 1.1 \\
\hline Distancia (m) & 100 & 100 & 100 \\
\hline
\end{tabular}

* 01 dólar americano $=25$ pesos cubanos.

producción de 80 metros cúbicos. Valorando los precios actuales del petróleo y la situación de la economía cubana, este ahorro de energía es el mayor aporte de este trabajo.

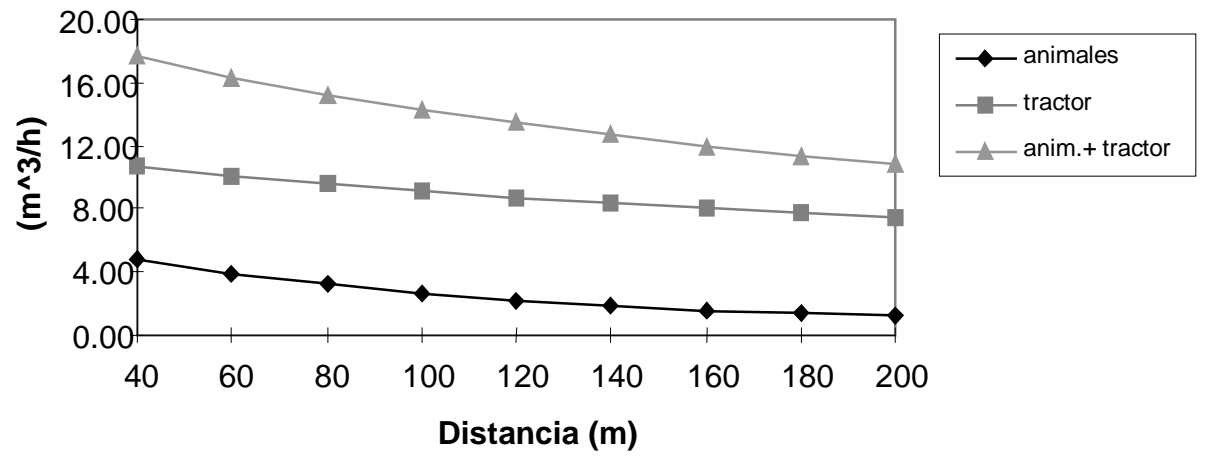

Figura 4 - Rendimientos en función de las distancias de arrastre. Figure 4 - Yield as function of the pulling distances. 


\subsection{Análisis de los rendimientos para los métodos evaluados}

La Figura 5 muestra la disminución de los rendimientos con el aumento de las distancias de arrastre. Para una distancia promedio de $100 \mathrm{~m}$, los animales produjeron $2.65 \mathrm{~m}^{3} / \mathrm{h}$ efectiva de trabajo, mientras los tractores alcanzaron un rendimiento de $9.54 \mathrm{~m}^{3} /$ h, con una diferencia de $6.89 \mathrm{~m}^{3} / \mathrm{h}$. En la Figura 5 se aprecia que, a los $100 \mathrm{~m}$, los animales consumieron menos tiempo para completar el ciclo que los tractores, la diferencia en el rendimiento se explica por el alto volumen por ciclo que extrajo el tractor, $2.25 \mathrm{~m}^{3}$, con respecto a $0.36 \mathrm{~m}^{3}$, extraído por los animales.

Considerando que los animales no pueden trabajar con igual ritmo, ni la cantidad de horas diarias que los tractores y, analizando los resultados mostrados anteriormente, se puede deducir que, por cada tractor se necesitarían alrededor de 12 yuntas de bueyes para mantener los rendimientos diarios actuales de 30 metros cúbicos por jornada. De ahí que el uso del método 1 sería casi imposible en las condiciones de las empresas forestales de Cuba, debido a la cantidad de animales necesarios.

El método 3 se mantuvo para cualquier distancia de extracción como el de mayor rendimiento, con un volumen por ciclo de $2.58 \mathrm{~m}^{3}$.

Esta diferencia en el volumen con respecto al método 2, fue debido a la mayor carga del tractor, 10.3 árboles, en el método 3, aumentando el volumen. También la reducción del tiempo del ciclo de trabajo, en particular, el tiempo de carga para el método 3, en aproximadamente el 40\% (Tabla 1), repercutió significativamente en el aumento del rendimiento en el arrastre de madera.

\subsection{Análisis de las alteraciones provocadas a la cubierta vegetal del suelo}

En el examen realizado a la cubierta vegetal del suelo se determinó un cubrimiento total en las 15 ha evaluadas en su estado natural, después de realizada la chapea del bosque dentro del área. Una capa de 5.7 cm de espesor como promedio cubría el suelo, predominando acículas secas de pinos y, en menor proporción, restos de hojas de otras especies asociadas a los pinares.

Los resultados de la evaluación de los daños a la cubierta vegetal del suelo (Figura 6), para el arrastre con yuntas de bueyes, mostraron que el 6.25\% del área total sufrió alteraciones, provocadas fundamentalmente por la incidencia en el suelo de las puntas de los árboles arrastrados (Tabla 4).

Cuando se utilizó el tractor, las alteraciones en el área fueron del 17.11\%, y se debieron a la operación con el winche, además de otras alteraciones provocadas por el sistema de rodaje del tractor, sobre todo en los virajes dentro de las áreas de corte. El arrastre combinado logró reducir las alteraciones a 11.28\% con respecto a la utilización sólo del tractor, aunque lo más significativo fue la reducción de los daños severos en un $50 \%$.

Los animales, a pesar de haber entrado en el área una mayor cantidad de veces que los tractores, cau-

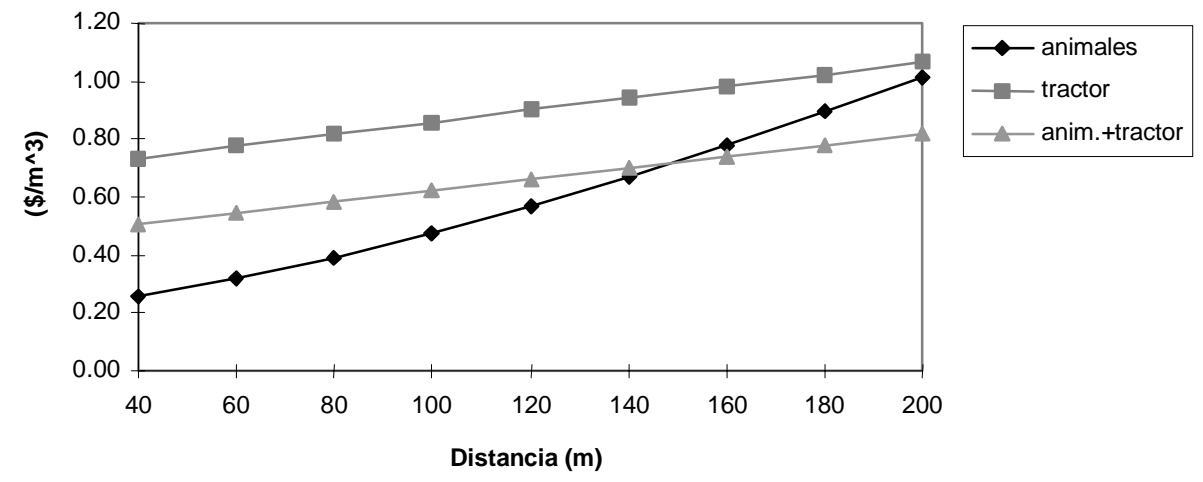

Figura 5 - Costos unitarios en función de la distancia de arrastre.

Figure 5 - Unit costs as function of the pulling distance. 


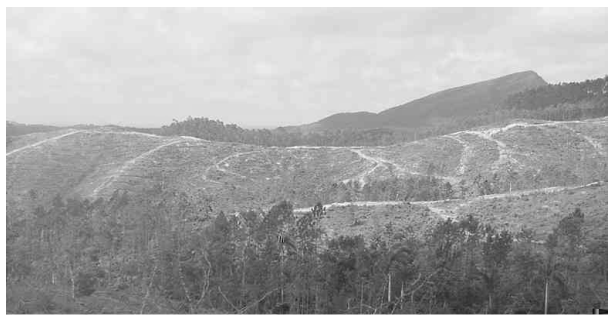

A

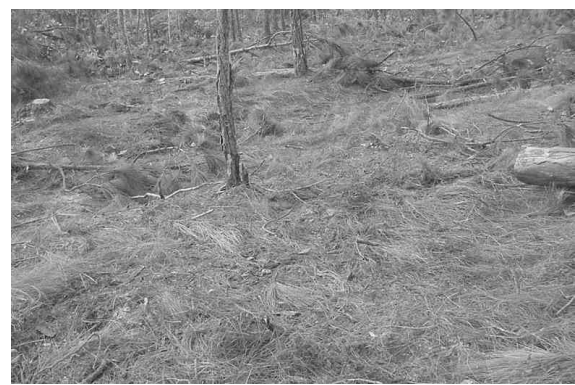

B

Figura 6 - A) Daños provocados al suelo por el arrastre de madera con tractor. B) Vista del área trabajada con animales.

Figure 6 - a) Soil damages caused by log pulling by tractor. b) View of the area worked by aninals.

Tabla 4 - Resultados de los daños causados por cada método de arrastre

Table 4 - Results of damages caused by each pulling method

\begin{tabular}{lccc}
\hline Tecnologías & $\begin{array}{c}\text { Superficie } \\
\text { total dañada } \\
(\%)\end{array}$ & $\begin{array}{c}\text { Superficie } \\
\text { con daños } \\
\text { severos }\end{array}$ & $\begin{array}{c}\text { Número de } \\
\text { ciclos } \\
\text { por ha }\end{array}$ \\
\hline Yunta de bueyes & 6.25 & 1.76 & 289 \\
Tractores & 17.11 & 12.23 & 45 \\
Bueyes + Tractores & 11.28 & 6.16 & 39 \\
\hline
\end{tabular}

saron daños mucho menores. El porcentaje de daños severos también fue más bajo. Estos resultados coinciden con los obtenidos por Cordero (1995) en la extracción de madera en Costa Rica.

\section{CONCLUSIONES}

De los resultados obtenidos en la investigación se deriva:

- El tiempo del ciclo de trabajo de los animales fue inferior al de los tractores, a pesar de la mayor velocidad de estos últimos, debido al excesivo gasto de tiempo requerido en la carga y descarga de los árboles de los tractores.

- La combinación de animales y tractores para el arrastre de los árboles logró disminuir considerablemente el tiempo del ciclo de trabajo con respecto al tractor, aumentar el volumen de madera por ciclo $\mathrm{y}$, por supuesto, mejorar los rendimientos en el arrastre de madera para cualquier distancia, evitando pérdidas de tiempo en el traslado de los animales a largas distancias y en la carga de los árboles para el tractor.

- El método 3, combinación de animales y tractores, fue la variante más económica para distancias de arrastre superiores a 150 m, que son las más empleadas con el fin de evitar costos con la construcción de caminos y puntos de carga. Esta opción permitió un ahorro de 0.4 litro de combustible por metro cúbico de madera extraída, cuando comparada con el método 2 .

- A pesar de que los animales realizaron más recorridos dentro del área, los daños que provocaron fueron pequeños con respecto a los métodos donde se empleó el tractor. Además, el tractor produjo mayor porcentaje de daños severos que los animales, aunque la combinación de animales y tractores redujo a 50 \% estos daños.

\section{REFERENCIAS BIBLIOGRAFICAS}

CHIRGWIN, J.C. Los animales de trabajo y sus múltiples aportes al desarrollo agrícola y rural. Roma: FAO, División de Producción y Sanidad Vegetal, 1994. 13 p.

CORDERO, W. Uso de bueyes en operaciones de aprovechamiento forestal en áreas rurales de Costa Rica. Roma, FAO, 1995, 33 p. (Estudio monográfico de explotación forestal).

FENNER, P. Relación entre el tráfico de vehículos y las deformaciones físicas de los suelos forestales. In: SEMINÁRIO DE ATUALIZAÇÃO EM SISTEMAS DE COLHEITA DE MADEIRA E TRANSPORTE FLORESTAL, 9., 1996, Curitiba. Anais... Curitiba: UFPR/FUPEF, p. 39-44, 1996.

GRÁ, H. et al. Tabla dasométrica de Pinus caribaea. Instituto de Investigaciones Forestales. La Habana: 1990. 28 p. (Informe Técnico). 
HARVEY, M. Rapport des essais de debusquage conventionnel dans les pentes avec des pneus larges. Projet de pneus. ballons 931-86-051. Quebec: Ministere de Energia et des Ressources Gestion Hauterivee, 1986. 18 p.

HENDRISON, J. Damage-Controlled Logging in Managed Tropical Rain Forest in Suriname. The Netherlands: Agricultural University Wageningen. 1990. p. 62-138.

HERNÁNDEZ, A. et al. Nueva versión de clasificación genética de los suelos de Cuba. La Habana: Ministerio de la Agricultura, Instituto de suelos de Cuba. 1994.

\section{INSTITUTO NACIONAL DE RECURSOS} HIDRÁULICOS INRH. Informe sobre registros climáticos. $P R$ 159. La Pimienta y PR 434. La Amistad. San Andrés. La Palma. Pinar del Río, 1996. 11 p.

MINAGRI. Dinámica de manejo de los bosques de coníferas. Pinar del Río: Servicio Estatal Forestal. Ordenación de Montes, 1996. 14 p.

NORMA CUBANA 34-38. Máquinas

agropecuarias y forestales: metodología para la evaluación económica. La Habana: 1985. 8 p.
SARRE, A. Extracción por cable, aumento o reducción del daño causado al bosque?

Actualidad Forestal Tropical, v. 1, n. 2., p. 3-5, 1993.

SESSIONS, J. Cost Control in Forest Harvesting and Road Construction. Forestry Paper, n. 99, p.106, 1992.

SEIXAS, F.; RUMMER; R.; STOKES, B.J. Forest harvesting in the United States: a search for sustainable management in balance with the ecosystem. In: SEMINÁRIO DE ATUALIZAÇÃO EM SISTEMAS DE COLHEITA DE MADEIRA E TRANSPORTE FLORESTAL, 9., 1996, Curitiba. Anais.... Curitiba: UFPR/FUPEF, 1996. p. 73-94, 1996.

VOLPATO, C.E.S.; MACHADO, C.C.; SOUZA, A.P. Otimização da produtividade e do custo da extração florestal com guincho arrastador. In: SIMPÓSIO BRASILEIRO SOBRE EXPLORAÇÃO E TRANSPORTE FLORESTAL, 1., 1991, Belo Horizonte. Anais.... Belo Horizonte: SIF/UFV. p. 113-126.

WARKOTSCH, P.W.; OLSEN, G.F. Identification and quantification of soil compaction due to various harvesting methods. In: ANIMAL MEETING OF THE COUNCIL OF FOREST ENGINEERING. IUFRO, 17., 1992. Oregon: 1992. 16 p. 